\title{
Ferromagnetic Resonance in Cast Microwires and its Application for The Non-Contact Diagnostics
}

\author{
SA Baranov* \\ Institute of Applied Physics, Academiei str. 5, Chisinau, MD-2028 Republic of Moldova
}

*Corresponding author: SA Baranov, Institute of Applied Physics, Academiei str. 5, Chisinau, MD-2028 Republic of Moldova.

Received Date: May 22, 2020

Published Date: June 09, 2020

\begin{abstract}
The natural ferromagnetic resonance reveals large residual stresses appearing in the microwire core in the course of casting. These stresses, together with the magnetostriction, determine the magnetoelastic anisotropy. Beside the residual internal stresses, the natural ferromagnetic resonance frequency is influenced by external stresses applied to the microwire or to the composite containing the latter (the so-called stress effect).

The dependence of the natural ferromagnetic resonance frequency on the deformation of the microwires is proposed to be used in the distant diagnostics of dangerous deformations of critical infrastructure objects
\end{abstract}

Keywords: Cast glass-coated amorphous magnetic microwire; Magnetostriction; Natural ferromagnetic resonance

\section{Theory}

The properties of the magnetic glass-coated cast amorphous micro and nanowires studies in many publications by various research groups [1-21]. The phenomenon of natural ferromagnetic resonance (NFMR) in the magnetic glass-coated cast amorphous micro and nanowires [4,6-13] is extremely interesting from the viewpoint of using it for non-contact diagnostics of distant of critical infrastructure objects.

The diagnostics become possible due to the stress effect on the NFMR, that is, the shift of the resonance frequency as a result of a deformation of the object. Such a frequency shift can be measured by irradiating the object with microwaves emitted by radar at frequencies near the NFMR and detecting the reflected signal, thus revealing a deviation of the resonance frequency from the original value.

The glass coating of the cast GCAMNWs induces strong mechanical stresses in the kernel [4]. In cylindrical coordinates, the residual tension is characterized by the axial, $s_{\mathrm{z}}$, radial, $s_{\mathrm{r}}$, and tangential, $s_{\varphi}$, components. The value of these stresses depends on the ratio of the radius, $\mathrm{R}_{\mathrm{m}}$, of the metallic kernel to the total microwire radius, $\mathrm{R}_{\mathrm{c}}$ :

$$
x=\left(\frac{R_{c}}{R_{m}}\right)^{2}-1,(1)
$$

Using the cylinder-shell model, we then obtain a formula for stresses in the metallic kernel of the cast GCAMNWs:

$$
\begin{gathered}
\sigma_{r}=\sigma_{\phi} \equiv P_{v} \\
P_{v}=\varepsilon E_{1} \frac{k x}{[k(1-2 v)+1] x+2(1-v)}
\end{gathered}
$$

where $\varepsilon E_{1}=\sigma_{0} \sim 2 \mathrm{GPa}$ is the maximum stress in the metallic kernel; $\varepsilon$ is the difference between the thermal expansion of the metallic kernel and that of the glass shell with the expansion coefficients $\alpha_{1}$ and $\alpha_{2}: \varepsilon=\left(\alpha_{1}-\alpha_{2}\right)\left(T^{*}-\mathrm{T}\right)$; is the Young modulus of the metallic kernel, $\mathrm{T}^{*}$ is the solidification temperature of the composite in the metal /glass contact region ( $\left.\mathrm{T}^{*} \sim(800 \ldots 1200) \mathrm{K}\right), \mathrm{T}$ is the room temperature; $\mathrm{k}$ is the ratio between Young's moduli of the 
glass and the metal ; $v$ is the Poisson ratio. Let us consider the case where all the Poisson ratios are $v=1 / 3$ in order to obtain

$$
\begin{aligned}
& P=\varepsilon E_{1} \frac{k x}{(k / 3+1) x+4 / 3}, \\
& \sigma_{z}=P \frac{(k+1) x+2}{k x+1} .
\end{aligned}
$$

With additional longitudinal strain, which occurs when the microwire is embedded in a solid matrix that itself deforms under external influence, the following term is added to the expression for the residual axial tension:

$$
\sigma_{e z}=\frac{P_{o}}{S_{m}(k x+1)},
$$

where $\mathrm{P}_{\mathrm{o}}$ is the force applied to the composite; $\mathrm{S}_{\mathrm{m}}=\pi\left(\mathrm{R}_{\mathrm{m}}\right)^{2}$ is the microwire cross-sectional area.

The theory of NFMR is presented in Refs. [6-8]. The NFMR frequency can be written as

$$
\omega(G H z) \approx \omega_{o}\left(\frac{0.4 x}{0.4 x+1}+\frac{\sigma_{e z}}{\sigma_{o}}\right)^{1 / 2},
$$

$$
\omega_{o}(G H z) \approx 1,5\left(\mathbb{( 1 0}^{6} \lambda\right)^{1 / 2}
$$

where

\section{Conclusion}

We have presented simple analytical expressions for the residual and external stresses in the metallic kernel of the microwire, which clearly show their dependence on the ratio of the external radius of the microwire to the radius of the metal kernel and on the ratio of Young's modules of glass and metal. The NFMR phenomenon observed in glass-coated magnetic microwires opens up the possibility of developing new materials with a wide range of properties [19-21]. An important feature of cast microwires with an amorphous magnetic core is the dependence of the NFMR frequency from the deformation (see Equations (7)).

Therefore, this effect can be used for contactless diagnostics of deformations in distant objects (including critical infrastructures) reinforced by cast magnetic microwires with the stress effect. These objects are periodically scanned with floating-frequency radar to determine the deviation of the initial NFMR frequency due to potentially dangerous deformations of the monitored object.

Another principle of detecting mechanical strain is examined in Ref. [21]. This principle is based on the giant magnetoimpedance (GMI) effect. The GMI effect $[17,18]$ demands external magnetization of the sample which is not required in the NFMR method [6-8].

\section{Acknowledgment}

This work was supported by the Moldavian national project No. 09.3.3-LMT-K-712-08-0003.

\section{Conflict of Interest}

No conflict of interest.

\section{References}

1. GF Taylor (1924) A Method of Drawing Metallic Filaments and a Discussion of their Properties and Uses. Phys Rev 23: 655.

2. M Vazquez (2007) Handbook of Magnetism and Advanced Magnetic Materials. (Eds: H Kronmuller, S Parkin), John Wiley and Sons, New York, NY, USA 4: 2193-2226.

3. M Vazquez, AP Zhukov, J Magn (1997) Magnetic properties of Fe-based glass-coated microwires. Magn Mat, 170(3): 323-330.

4. SA Baranov, VS Larin, AV Torcunov (2017) Technology, Preparation and Properties of the Cast Glass-Coated Magnetic Microwires. Crystals 7(6): 136.

5. AS Antonov, VT Borisov, OV Borisov, AF Prokoshin, NA Usov (2000) Residual quenching stresses in glass-coated amorphous ferromagnetic microwires. J Phys D: Appl Phys 33(10): 1161.

6. SA Baranov (1998) Use of a microconductor with natural ferromagnetic resonance for radio-absorbing materials. Tech Phys Lett 24: 549.

7. SA Baranov, M Yamaguchi, KL Garcia, M Vazquez (2008) Current literature in mass spectrometry. Surf Engin Appl Electrochem 44(1): 245 .

8. SA Baranov (2015) Preparation of the cast glass-coated amorphous magnetic microwires. Moldavian J Phys Sci 14: 201.

9. P Marín, D Cortina, A Hernando, J Magn (2005) Fabrication of Ferromagnetic Wires. Magn Mater 290-291: 1597-1600.

10. HX Peng, F Qin, MH Phan (2016) Ferromagnetic microwires composites: from sensors to microwave applications, Springer, Switzerland, pp. 1214.

11. F Qin, HX Peng (2013) Ferromagnetic microwires enabled multifunctional composite materials. Progr Mater Sci 58: 181.

12. L Kraus, G Infante, Z Frait, M Vázquez (2011) Ferromagnetic resonance in microwires and nanowires. Phys Rev B 83: 174438.

13. L Kraus (1982) Catalytic Hydrazination. Czech J Phys 32: 264.

14. 0 Reynet, AL Adenot, S Deprot, O Acher M Latrach (2002) Effect of the magnetic properties of the inclusions on the high-frequency dielectric response of diluted composites. Phys Rev B 66: 094412.

15. SN Starostenko, KN Rozanov, AV Osipov (2006) Microwave properties of composites with glass coated amorphous magnetic microwires. J Magn Magn Mater 298: 56.

16. F Yıldız, BZ Rameev, SI Tarapov, LR Tagirov, B Aktaş, et al. (2002) Study of Microwave Magnetoimpedance Effect in Amorphous Fesib Wires. Magn Magn Mater 247: 222.

17. D Ménard, M Britel, P Ciureanu, A Yelon (1998) Giant magnetoimpedance in a cylindrical magnetic conductor. J Appl Phys 84: 2805.

18. D Ménard, AYelon (2000) Oxygen vacancies in ZnO. J Appl Phys 88: 379.

19. MG Nematov, AM Adam, LV Panina, NA Yudanov, A Dzhumazoda, et al. (2019) Evolution of the magnetic anisotropy and magnetostriction in Co-based amorphous alloys microwires due to current annealing and stress-sensory applications. J Magn Magn Mater 474: 296.

20.SA Baranov (1999) Generalized conductivity and optimum energy release. Tech Phys 44: 853.

21. D Makhnovskiy, A Zhukov, V Zhukova, J Gonzalez (2008) Tunable and SelfSensing Microwave Composite Materials Incorporating Ferromagnetic Microwires. Adv Sci Technol 54: 201-210. 